\title{
PAW - TOWARDS A PHYSICS ANALYSIS WORKSTATION
}

\author{
R. BOCK *, R. BRUN *, O. COUET **, J.C. MARIN *, R. NIERHAUS *, L. PAPE *, \\ N. SAUMON **, C. VANDONI * and P. ZANARINI * \\ * CERN, 1211, Geneva 23, Switzerland \\ * * LAPP, Annecy, France
}

\begin{abstract}
In recent years interactive data analysis and presentation packages have become more and more attractive on conventional timesharing systems. The advent of personal workstations with their good response time and powerful graphics capabilities is making these systems even more popular. PAW has been designed in that spirit. It combines the best features and the experience acquired with the existing systems together with the user friendliness now possible on modern workstations. Rather than being a monolithic system, which may be well suited to one operating system, PAW is built with modules which can also be used in the batch environment or by other interactive programs. PAW has been designed to work on many different configurations.
\end{abstract}

\section{Data analysis today and PAW objectives}

At present, standard event analysis for most experiment ends with the creation of DST-s (data summary tapes). From there on, physicists resort to packages that allow the creation of histograms usually identified by single integer numbers, and their sequential storing by the local system's file manager for later access. Few physicists with access to the right kind of hardware make use of histogram manipulation packages, but even then the most common output of histograms is on the line printer or consists of inadequate hardcopies from passive terminals. For informal presentations, copies of line printer output with hand annotations are commonplace. Publishable graphs are hand produced, typically in a drawing office. There is now no accepted way of keeping large numbers of histograms in a manageable structure, accessible to groups of researchers, with additional semiautomated comment and date fields for ease of documentation.

The increasing success encountered in recent years by interactive data analysis and presentation systems such as HTV [1] at CERN, GEP [2] at DESY or IDA [3] at SLAC on conventional timesharing systems is significant of a new trend towards interactive computing. The Physics Analysis
Workstation package PAW[4] was launched at CERN in 1986. It is now in its completion phase. Intended as a replacement for HTV, it provides more facilities both in the data presentation and in the data analysis parts. Particular emphasis has been put on the quality of its user interface. The package is implemented on the most commonly used timesharing systems, but its best use will be on personal workstations which are becoming more and more popular in all fields of computing.

For this kind of interactive work, Personal Workstations with a capacity of 1 Mips * are considered more cost effective than big mainframes of 30 Mips supporting 300 users or superminis of 4 Mips supporting 40 users. It is now possible to buy a well equipped workstation with 4 megabytes of main memory and local storage of 150 megabytes for less than $\$ 20000$. Present-day computer host systems with connected personal workstations call for an adaptation of working style. Workstations have brought graphics interfaces that are easy to deal with even for the casual user. File storage, accessible to central and distributed computers, can be used for accumulating histograms with common access for many physicists, and with proper documentation appended. Workstations

\footnotetext{
* Million Instructions Per Second.
}

0010-4655/87 $\$ 03.50$ () Elsevier Science Publishers B.V. (North-Holland Physics Publishing Division) 
with their graphics screen can then be used to choose from the histogram base those combinations that show most clearly the intended physics message, and to settle on their best presentation parameters. Finally the small number of publishable graphs can be edited until true publication quality is obtained. A high quality device, possibly central and network-connected, will allow physicists to produce their plots directly and on their own timescale, without having to wait for an overworked drawing office. As all of these steps can be repeated if properly recorded as macro instructions, changes in input data can smoothly be propagated into the final graphics output with minimal work. The data analysis chain can be divided into 3 main steps.

\subsection{Step 1}

Step 1 is executed by a group of service routines to be called by a FORTRAN program (typically a DST analysis job) and providing facilities to create statistical data (histograms in 1 or 2 variables, tables, $\mathrm{N}$-tuples). These routines are very close to the existing successful HBOOK package [5], run on a computer centre or local host computer. HBOOK has been amended by various facilities mostly proposed by its many users, and most prominently by a substantial improvement in the generality of its output streams. Facilities to structure and tag the created objects to ease later comparative analysis of similar histograms have been included into a new version. A simple hierarchy of output and the possibility to accumulate histograms produced in several jobs in a single file results in a histogram data base. This base together with a suitable identification scheme then give access to the output of many jobs to a group of collaborating physicists, avoiding the usual problems of communicating private file name conventions and of histogram identifier clashes. The possibility of a standard output facility for extremely dense micro-DSTs(N-tuples) with limited resolution in variables, for later selection upon passing again in a histogramming step, is also part of the new version. Such substitutes for DST-s can then be kept locally on disk and will undoubtedly make the workstation more efficient for frequent changes in cuts, selection criteria etc.

\subsection{Step 2}

Step 2 is the heart of PAW: an interactive histogram manipulation program, to run on a workstation or on a local host (even with unintelligent graphics terminals), depending on availability, with easy access to the file base generated in step 1. Its objective is the comparative analysis of statistical plots and a choice of presentation. Editing possibilities for the resulting graphics file (adding of text, choice of line style, fonts etc.) are also available. Portability of the program and of the resulting graphics files is considered essential. The package may be driven by commands or by menu with command backup, and contain possibilities for storing command sequences permanently as macros. Graphics output is in portable form, and ample storage facilities for graphics output exist, possibly by shipping files to a host machine with large file store and backup. We also have taken into account the needs of experiments who may want to use this package in a real-time environment.

\subsection{Step 3}

Step 3 finally is an interactive program for the manipulation of graphical information, implementable only on a workstation with modern graphics facilities (bitmap display). This program allows a user to transform graphical presentations into publishable diagrams. Different fonts, area filling, line style, zooming, moving and duplication of picture parts etc. are the relevant functions in this program. General iterative editing (change of style, addition, deletion of comments or picture parts) is possible, and high quality hard copy output facilities are essential. Access to graphics files created in step 2 (which may, of course, also run concurrently), and facilities for storage of the resulting graphics output must be available. The transfer of graphs into a text processing system is the last stage of the interaction data analysis chain.

\section{The structure of the PAW system}

\subsection{The PAW modules}

PAW is based on modules, described in the following sections, which can also be used inde- 


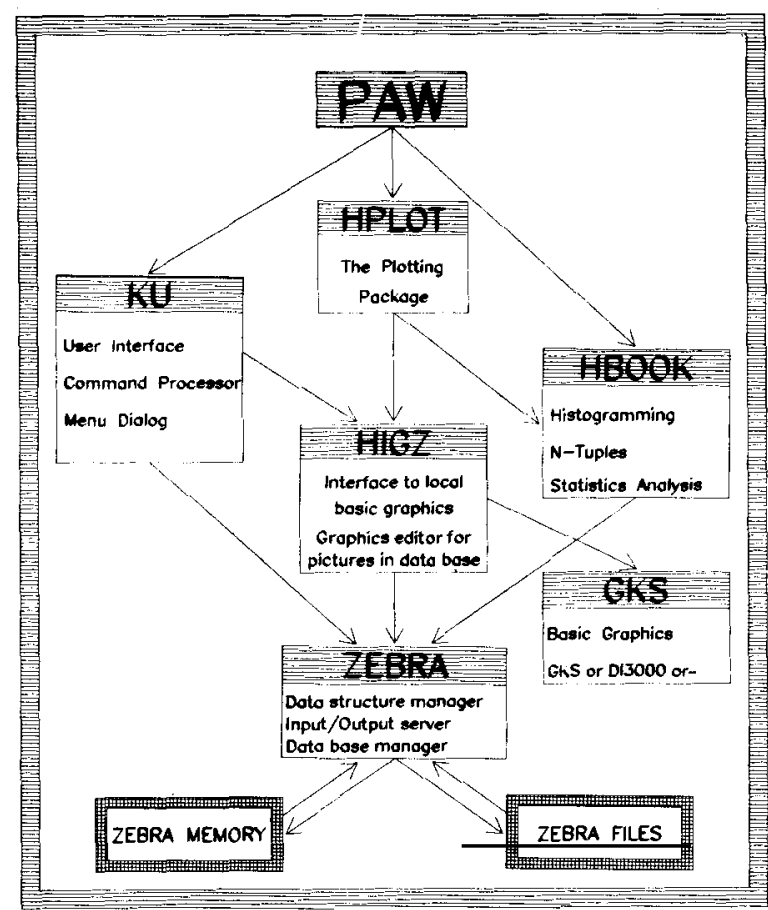

Fig. 1. PAW Structure. The various modules of the system and their relationship.

pendently either in batch mode or interactively. This is particularly true for the histograming module HBOOK [5], the graphics package HIGZ [10], the data structure management system ZEBRA [8] and the user interface package KU [9]. The general organization of the system is shown in fig. 1.

All these modules are organized as a library and can be called from any user application program. An executable module, called PAW, can be invoked in an interactive session with the possibility to read user profiles or startup files. The user has also the possibility to call the top-level routine of PAW, to link it with his own application (reconstruction program, etc.), perhaps adding some additional commands.

\subsection{The PAW objects}

PAW deals with objects of different types and with different attributes. We consider as vital the possibility to store and address these objects in a coherent form, as part of a multi- or single-file data base. The definition of objects and their identification:
Macros are sequences of PAW commands, typically obtained in a PAW session by the $\mathrm{M} /$ Edit_Create or $\mathrm{M} / \mathrm{Get}$. Log commands. They are stored as character vectors, with a line length of 80 characters.

Vectors are arrays of varying information content, most frequently of floating point numbers. In general, they are simply collections of elements of the same type. Floating point and integer vectors have a number of supporting operations associated. The implementation of an array manipulation language derived from SIGMA [7] is foreseen. Vectors may be generated by an independent FORTRAN job and stored by a call to the ZEBRA routine RZVOUT. Most frequently, vectors are generated explicitly in a PAW session.

Functions are sequences of one or more statements of FORTRAN-like syntax. PAW functions produce a single function value as result, they may have one or more parameters, and they have access to PAW vectors both for input and output.

Histograms are objects containing accumulated information. They are generated in HBOOK or PAW from event data, using typically a single definition call ('booking'), and multiple 'filling' calls for entering events. They may also be derived from other histograms by histogram operations (e.g. in a PAW session). Histograms represent event frequencies as function of a single variable (1D histograms), or as function of two variables (2D histograms). 2D histograms may have attached one or more projections by integrating over one of the two variables. The information of histograms includes the title, the bin and packing definition, the bin contents and bin errors, statistics values, possibly an associated function vector, and output at- 
tributes. Some of these items are optional.

$\mathrm{N}$-tuples are objects containing single-event information of a fixed length, for any number of events. The number of events, the number of words per event, and the per-event information make up the information of an N-tuple. N-tuples can be transformed into subsets by selection and transformation of content, and can be statistically analyzed by producing histograms. The system is fast enough to be used interactively inside PAW and thereby giving to the physicists a formidable tool for interactive data analysis. The $\mathrm{N}$-tuple is a new facility in HBOOK4. Although quite different in implementation the idea is very similar to the approach of GEP[2].

Graphics are objects composed of graphics structures primitives, and corresponding to the graphical presentation of objects in a viewport. Graphics Structures are generated by the HIGZ graphics interface.

\subsection{PAW object names}

Any object addressable in a PAW session carries a unique full name decomposed as follows:

- The simple proper name is a string of alphanumeric characters (underscore included). Objects generated in a PAW session are frequently called by their proper name alone, making use of the current working directory facility. In the case of objects created by HBOOK the proper name is a non-zero integer. The proper name may also be composite, if a qualifying name is appended using the full stop $\because$ as separator. The qualifying name applies to objects derived from and dependent on other objects. A projection of a 2D histogram, itself a 1D histogram, will be named by the parent histogram's name with a qualifying name appended.

- The path name is a necessary extension of proper names for identification of groups of objects. It precedes the proper name by concatenation, and corresponds to the directory in which the object is stored. A minimal path name consists of a top directory only, which may characterize the local PAW temporary store, or the external file on which the object is stored. More complex path names result from structuring directories further.

- The cycle number is a largely automatic option allowing the storage of different versions of an object stored with the same proper name in the same directory. An object stored with a name already present, will automatically be given a name with the cycle number incremented by one. When referring to an object without indicating the cycle, the most recent one (highest cycle number) will be selected. Reference to individual cycles is possible.

The full name of an object is obtained by concatenating, from the left, the path name (i.e. the name of the directory), the simple proper name with the qualifying name if any, and the cycle number. A slash $(/)$ separates the path name from the proper name, a full stop (.) separates the simple proper name and qualifier(s), a semicolon (;) separates the cycle number from the proper name. The full name of an object 'Nobj' in a directory 'Top/Sub', with qualifying name 'Qual' will then read:

Top/Sub/Nobj.Qual;Cycle

Examples:

- All_data_uncut/Calorimeters/Electromag/ 1024; 1

- Charm_Decays/154.Bany.5;1

- Session_05Sep/Xsection_Picture;2

The above notions map onto the ZEBRA RZ package: Proper names are keys in $R Z$, with the notion of (sub)directory and cycle identical to the RZ conventions. Any object may be referred to under its unabbreviated full name. In this case, the path name starts with a double slash $(/ /)$. Path name abbreviations can be obtained using either the Current Working Directory or a freely chosen Alias. 


\section{The data structure management system ZEBRA}

All of-line programming in high-energy physics is carried out, for various reasons, in FORTRAN77. While this language offers certain advantages over its competitors, it does suffer from one serious defect, namely its lack of dynamic structuring facilities.

It is to overcome these difficulties that the ZEBRA [8] system has been written. It allows not only a truly dynamic creation of data structures at execution-time, but also the added advantage of being able to manipulate those structures, and even to write them to an external medium and to recover them intact on some other computer.

ZEBRA manages any type of structure, but specifically supports linear structures (lists) and trees. In addition, ZEBRA permits Input/Output to either sequential or direct access files, depending on the nature of the data and, very important, it also provides two modes of data representation. The first is called native mode, and implies that the data undergo no conversion when transfered between storage and the external medium. Such data may be read only on a computer of a compatible architecture. The exchange mode, on the other hand, allows transfer of data between a large variety of computers by making appropriate conversions to and from an interchange format. The direct access package $R Z$ can be used to manage hierarchical data bases. The package also contains tools for describing the layout of banks and data structures, which allow the automatic generation of documentation.

ZEBRA provides a significant extension to the power of FORTRAN, at an insignificant cost in terms of execution-time overheads. Without its concepts, substantial time would be wasted in developing large programs. The system is now widely-spread in the High-Energy-Physics environment.

\section{The histograming and plotting packages $\mathrm{HBOOK}$ and HPLOT}

HBOOK [5] is a well known FORTRAN-callable histogramming facility, whose purpose is to
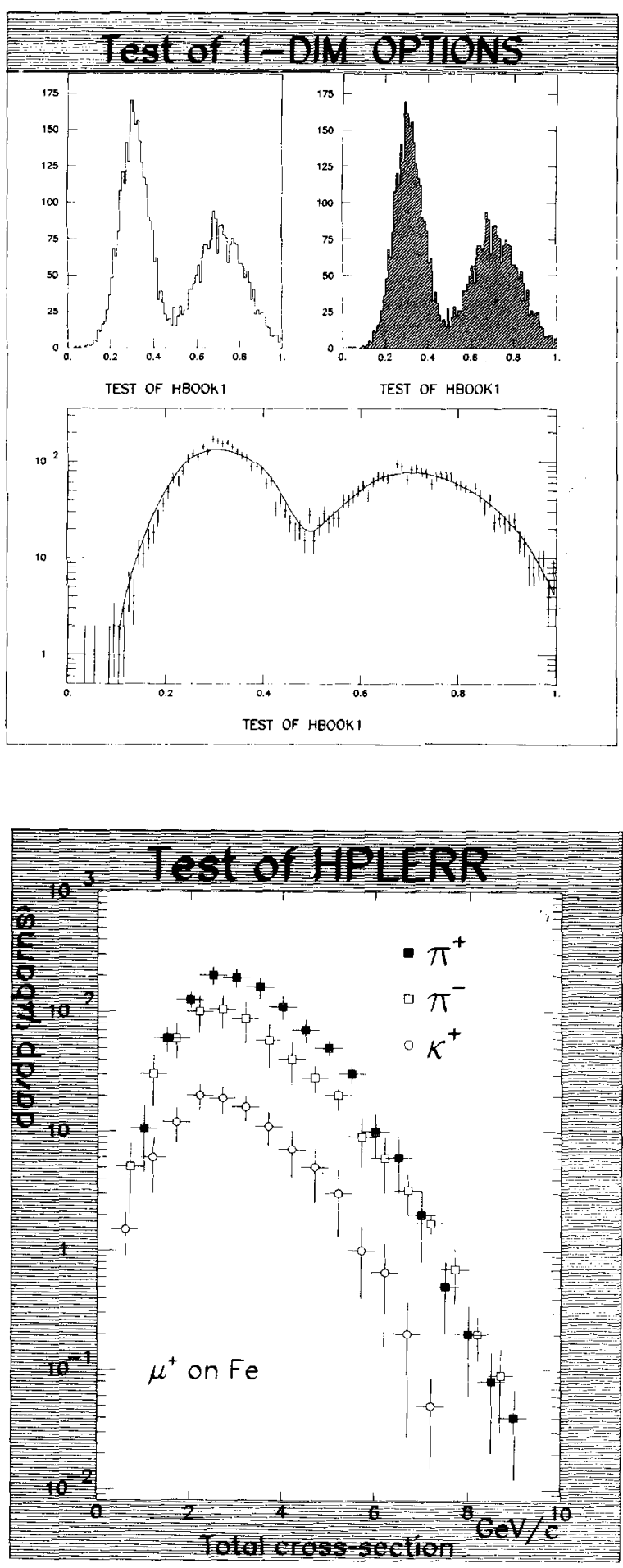

Fig. 2. 1-DIM options: shading, superimposed function, windowing. 


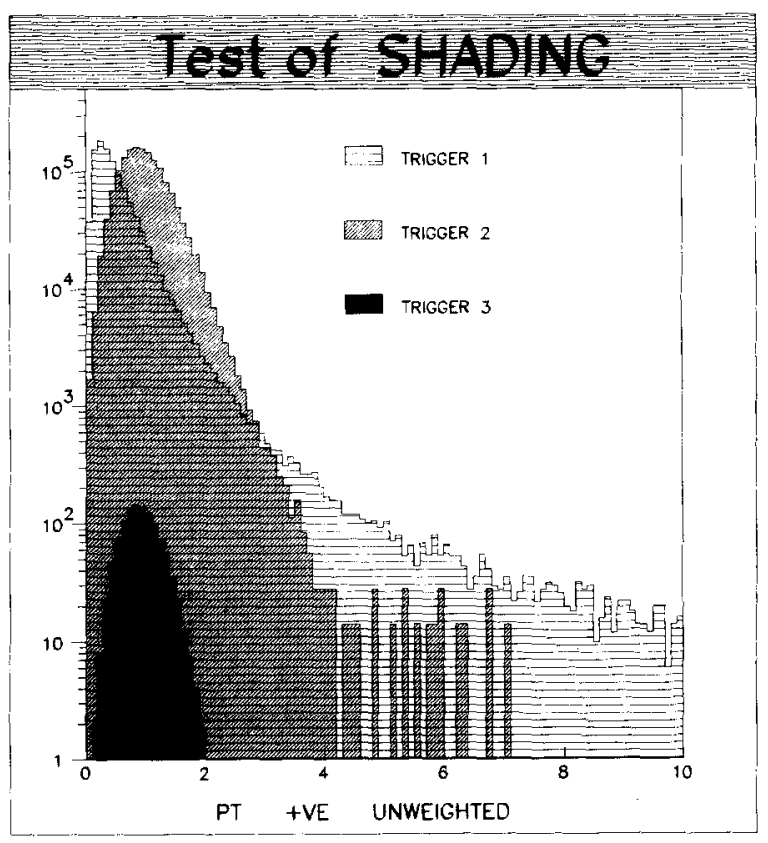

Fig. 3. LOG scale, symbols and annotations, interior styles.

define, fill, edit, and save histograms, scatter-plots and tables. The output is formatted for the printer, but a graphics interface is provided by the HPLOT [6] package. These packages have been used for many years by thousands of physicists, engineers, students, etc. in the world. New versions with new facilities have been developed in the context of PAW. They are both based on ZEBRA and provide the following functionality:

- 1-DIM, 2-DIM histograms and N-tuples;

- Projections and slices of scatter-plots;

- Access to the contents of histograms;

- Operations between histograms;

- Comparison of histograms;

- Minimization and parametrization tools;

- Random number generation;

- Histograms can be structured in memory (directories);

- Histograms can be saved onto direct access ZEBRA files;

- Wide range of graphics options:

- Normal contour histograms, shaded histograms, error bars (see fig. 2 and fig. 3).

- Smoothed curves and surfaces;

- Scatter-plots, lego-plots, contour-plots (see fig. 4);

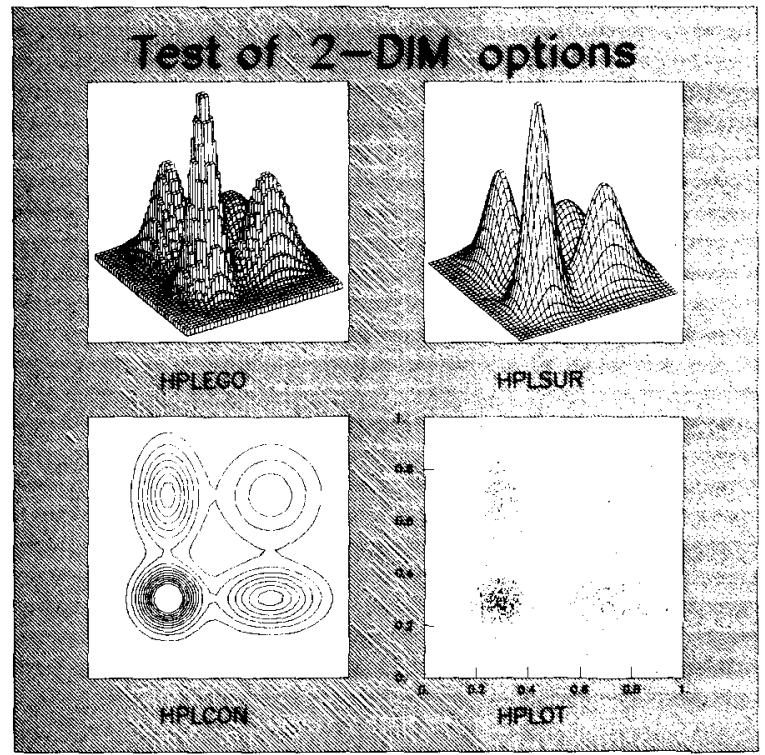

Fig. 4. 2-DIM options. lego-plot, surface, contour-plot, scatter-plot.

- Automatic windowing;

- Graphics input and basic graphics.

\section{The user interface system $\mathrm{KU}$}

A homogeneous user interface for the input and checking of commands and associated parameters is thought to be of vital importance. Because the requirements for experts and beginners are different, two ways of entering commands on the station are considered:

- by selection from menus. The menu presentation on the screen is very much device-dependent. PAW offers (see fig. 5) both alphanumeric and graphics menu layout. The alphanumeric panel is in general based on the local screen management system. The graphics panel is well suited for graphics applications. If a bitmap display is available, hardware raster operations are used to copy menu boxes from main memory to display memory.

- by direct input in a command window.

In addition, blocks of commands may be entered by invoking a macro, the rules for commands inside a macro being the same as for direct 


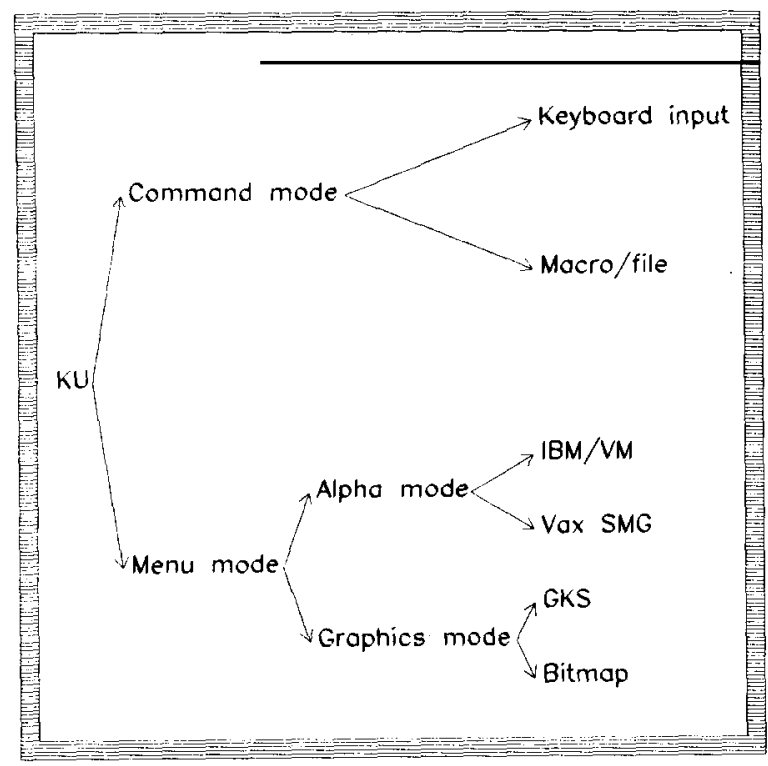

Fig. 5. The various $\mathrm{KU}$ Input modes.

input. An interface with the local text editor is provided for the creation or the modification of a macro. In both modes of user input, an unabridged and unambiguous command name must result so that macros can be build up from a session's log. Whereas command identification

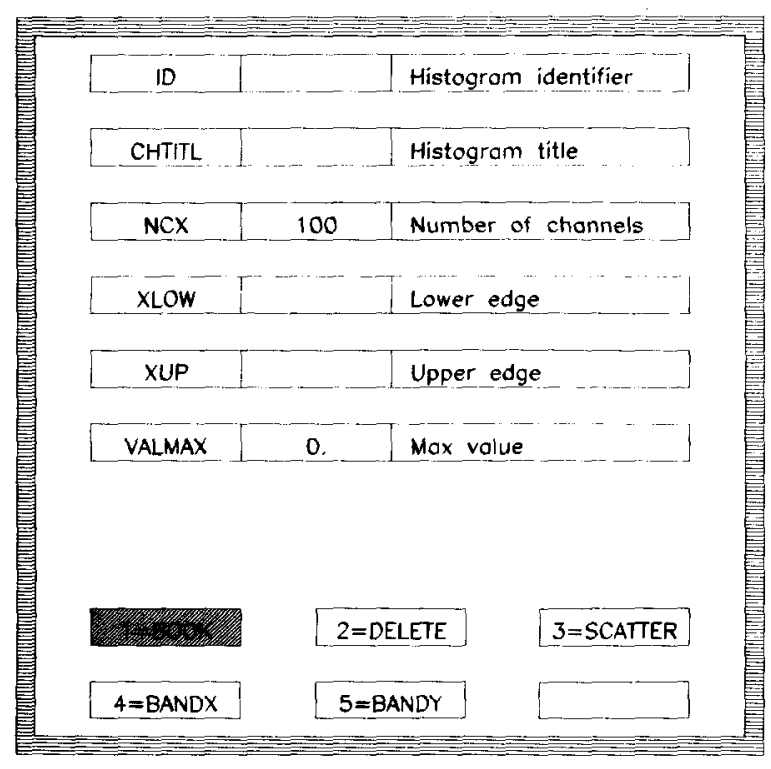

Fig. 6. An example of a full-screen dialogue. through a menu driven input is implicit, direct input must allow abbreviations for the expert user, and both unrecognized or ambiguous command names may result. Menus further attempt to guide a user by structuring commands into different levels. A full command name is the result of a suitable concatenation of these level names. It requires compaction or abbreviation for direct input.

The input of parameters associated to commands can be direct or prompted for in case of direct input; in menu driven operation a panel shows the various parameters and their defaults (see fig. 6).

The general structure for commands together with the code to be executed upon selection of one command is kept in an external file called the CDF (Command Definition File). From this file, the generation of all necessary internal command representations is possible. This unique and redundancy-free input will allow to interface with different command dialogues, and substantially ease the foreseeable evolution of the system in time. A more or less verbose documentation can also be derived.

\subsection{Command input}

\subsubsection{Command input by Menu}

A command prompted by menu is obtained by choices at different levels, each choice being characterized by an alphanumeric string that may contain underscores. The full command name is then defined by the concatenation of all such strings, levels being separated by slashes ' $\%$ ', e.g. Histogram/Operation/Add. This makes menu and command mode homogeneous.

\subsubsection{Direct command input by Typing}

The full command name as defined above is a unique but verbose command name. Abbreviations may be used and ambiguities are checked automatically. Further freely chosen abbreviation are possible which may still retain the original mnemonic value; they follow the same rules as for the names of PAW objects, and are defined in the following:

The lowest-level name is a valid abbreviation 
for a full name. If this abbreviation, as will often be the case, gives rise to ambiguities, one or more higher level names may be prefixed. In addition, any substring of the name contained between two separators / can be abbreviated by dropping letters from the right. By definition, characters in a command are not case sensitive, i.e. all are translated to upper case. In the following example two commands are abbreviated in various equivalent ways (leaving open the possibility of ambiguities).

File/Directory/Histograms

FIL/DIR/HIS

Hist (certainly ambiguous)

Dir/Hist

$\mathrm{F} / \mathrm{D} / \mathrm{H}$

Macro/Edit_Create

$\mathrm{MAC} / \mathrm{EDI}$

$\mathrm{M} / \mathrm{E}$

E_Create

\subsubsection{Input from Macro}

Whatever the input mode of commands is, the command processor keeps track of all executed commands in their standard abbreviation and with (checked) parameters appended, in the command log. The content of the log can be transformed into a macro by the Get_Log command, or a macro can be generated by a Edit _Create command. When the macro is invoked (by typing its name or selecting it on the menu), input control switches to the macro stack in direct command input mode. Control is returned to the command window or menu only after the stack has been emptied or a break sequence is executed.

\subsection{Parameter input}

Associated to commands may be lists of parameters, whose type and range may have restrictions associated. Parameters may be literals of various types or names of objects. A local dialogue results from parameters not satisfying the restrictions imposed, or from missing parameters. Parameters may have defaults.

\subsection{Local guidance}

Some additional guidance over direct input is provided by using a structured menu of command parts. Further short explanations beyond the mnemonic value of the menu items are provided for each command or command part as a standard feature. We call this permanently available option the command Guidance, as opposed to the Help facility which allows to access directly the full command documentation and other relevant parts of the User Guide. Guidance text is permanently associated to the command definition: with menu input, it is selectable at any level, while with direct input, a Guide command with a command name as parameter fulfils the same purpose.

\subsection{The command definition file}

Regardless which input mode is selected the definition of commands and their parameters as well as the associated guidance text must be given in a form we believe to be friendly and portable. It is easy to change, and allows the generation of command documentation and validity checking, like the absence of ambiguities when abbreviating. This representation of the command structure needs a transformation into a program-internal

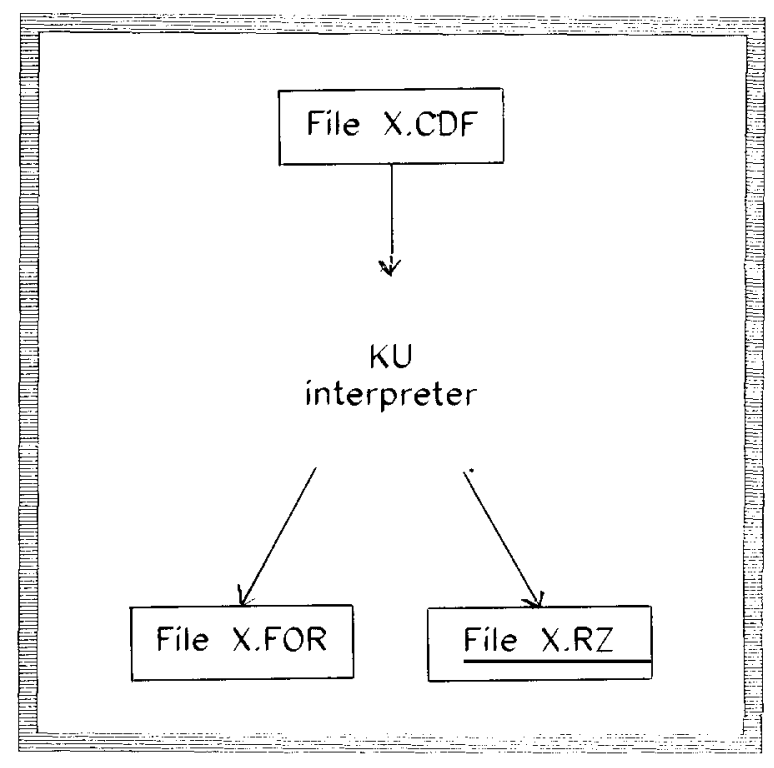

Fig. 7. The KU processor. Automatic generation of the Interactive program from the Command Definition file. The ZEBRA data structure of commands can be saved on a file in order to speed up the initialization phase of the interactive program. 
format, which is then used for menu presentation, generation of the command $\log$ and parameter checking (fig. 7). It contains, in addition to the elements of the full command name, the necessary parameters, their type and possibly a valid range and default values, the prompting instruction for each parameter, and the guidance text for each command or menu level. The file is structured for easy manipulation by the programmer, and formatted for simple interpretation and checking by a parsing routine.

\section{The graphics interface package HIGZ}

Graphics packages like GKS mediate the transition from user programs to devices in a standardized way. The European effort to restrict High Energy Physics users to using only one such package, GKS, will result in portability of application programs between systems on which GKS is installed, and will make the application programs largely device-independent.

These packages, however, have limitations. They do not foresee an acceptable way of recording large volumes of graphical information in compact form with a convenient access method for later manipulation. The GKS metafile is conceived as a vehicle to communicate series of pictures between computers and devices, but not for their later manipulation. Also, the acceptance of GKS, in particular by Laboratories outside Europe, is still rather modest, and thus it is not a standard that the High Energy Physics community can restrict itself to exclusively. We believe that the following requirements must be met by the graphical output of PAW:

- The PAW picture data base must be fully transportable.

- It must have easily accessible units (pictures) for later manipulation.

- The picture data must be as compact as possible, and accessible in direct access mode.

- The picture data must be independent from the underlying basic graphics package and, a fortiori, from different implementations of the same graphics package.

These requirements are not restricted to PAW.

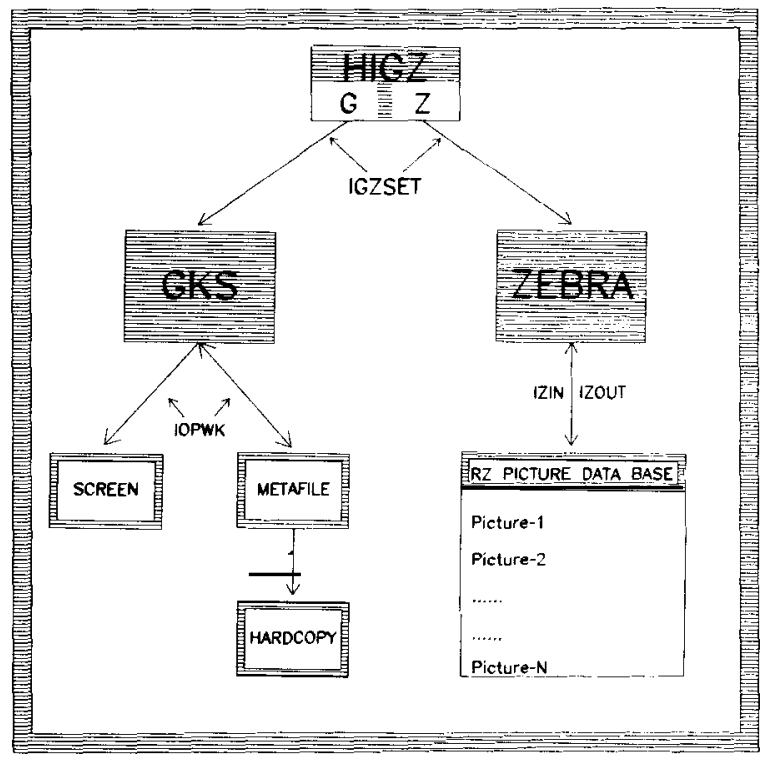

Fig. 8. HIGZ Structure. The GKS branch is a direct interface to the local graphics package. The ZEBRA branch allows the creation of 'pictures' that can be modified with the graphics editor.

They are common to many applications existing or under development. We have therefore designed an interface package called HIGZ [10] (for High level Interface to Graphics and ZEBRA), written in the context of PAW, and aiming at graphics applications of any nature, provided the level of functionality is similar. This package is basically a thin layer between the user program and the graphics package, offering the following advantages (fig. 8):

- an interface to a standard memory management system (ZEBRA), and though it is a mechanism to store graphics data in a way which makes their organization and subsequent editing possible and easy. The picture data base (with ZEBRA) is also highly condensed and fully transportable. A picture editor is part of HIGZ. It allows merging of pictures, editing of basic graphics primitives, operations onto HIGZ structures, etc.

- A GKS-like user interface to the graphics package, keeping the program independent of the graphics package installed.

The level of HIGZ was deliberately chosen to be 
as close to GKS and as basic as possible. This makes the interface to GKS a very simple one and preserves full compatibility with the most important underlying graphics package. HIGZ does not introduce new basic graphics features, and does not duplicate GKS functions. On the other hand some macroprimitives are proposed, providing very frequently used functions, such as circles and axis. The user will also be able to call GKS directly in parallel with the use of HIGZ. Interfacing HIGZ to other graphics packages is possible, but not as straightforward.

The system is separated into three main sets of functions:

- Basic graphics functions with calling sequences identical to those of GKS.

- Higher-level macroprimitives

- Memory management functions, interfacing to the memory management system (ZEBRA)

The user is able to invoke any of these sets of functions, simultaneously or not. This is particularly useful during an interactive session, as the user is able to 'replay' pictures previously created, with no need to recall the application program, but just accessing the picture data base.

\section{Conclusion}

The development of a large software system for a physicist workstation for data analysis has been presented. Such a station makes use of the modern workstation hardware now becoming popular in the High Energy Physics community, and is based as much as possible on existing software. General packages adaptable to different present or future hardware have been described. All are based on the idea of iterative interactive creation, hierarchical storage and comparative analysis of statistical information, with possibilities for high quality output. Graphics editing capabilities have been included. This package is expected to have considerable impact on that part of physics analysis which uses less CP time than event processing, but takes substantially more other resources on computer systems (tapes, disks, mass storage, etc.) and a much larger fraction of a physicists' time. In particular, care has to be taken to make analysis and graphics possibilities available to the non-expert physicist.

A partial release of PAW for $\beta$ testing is scheduled for the spring of this year and a general public release for the autumn.

\section{References}

[1] R. Brun, HTV Users Guide, CERN DD/EE/80-5.

[2] E. Bassler, GEP User manual, DESY 1985.

[3] T. Burnett, IDA Interactive Data Analysis system, SLAC MAC-III memo 1/83-6.

[4] PAW Users Guide, in preparation.

[5] R. Brun, D. Lienart, I. Ivanchenko and P. Palazzi, HBOOK Users Guide, CERN DD/EE/81-1.

[6] R. Brun, H. Renshall and H. Watkins, HPLOT Users Guide, CERN DD/US/17.

[7] C. Vandoni, SIGMA Users Guide, CERN DD/US/44.

[8] R. Brun, M. Goossens and J. Zoll, ZEBRA Users Guide, CERN DD/EE/85-6.

[9] KU Users Guide, in preparation.

[10] HIGZ Users Guide, in preparation. 